\title{
PRINCIPAIS FÁRMACOS UTILIZADOS NO TRATAMENTO DE DISLIPIDEMIAS: UMA ATUALIZAÇÃO
}

\author{
Karla Sunally Pereira de Sousa Siva ${ }^{1}$, Francisco Patricio de Andrade Júnior², \\ Júlia Beatriz Pereira de Souza* 3 \\ ${ }^{1}$ Farmacêutica pela Universidade Federal de Campina Grande. \\ ${ }^{2}$ Doutorando, Programa de Pós-Graduação em Produtos Naturais e Sintéticos Bioativos, \\ Centro de Ciências da Saúde, Universidade Federal da Paraíba, João Pessoa-PB. \\ ${ }^{3}$ Prof $\stackrel{\text { a }}{\text {. Dra }}$. Universidade Federal de Campina Grande, Unidade Acadêmica de Saúde, Centro \\ de Educação e Saúde, Cuité-PB. \\ Email para correspondência: juliabtriz@gmail.com
}

\section{Resumo}

As dislipidemias podem ser definidas como alterações nos níveis lipídicos da circulação, fazendo com que o uso de fármacos seja imprescindível para evitar o surgimento de problemas cardiovasculares. Objetivou-se realizar uma revisão da literatura sobre as principais classes de fármacos utilizados no tratamento das dislipidemias. Tratou-se de uma revisão da literatura nas bases de dados SciELO, BVS, MedLine e PubMed. O levantamento bibliográfico ocorreu no período de janeiro a junho de 2019. As estatinas inibem a etapa limitante na síntese do colesterol e são fármacos preferidos na hipercolesterolemia. Os fibratos, por sua vez, são considerados medicamentos de primeira escolha para a hipertrigliceridemia, enquanto que os sequestradores de ácidos biliares e a ezetimiba geralmente são usados em associação com outros hipolipemiantes no tratamento da hipercolesterolemia. A niacina modifica favoravelmente todas as principais frações lipídicas e é o fármaco mais eficaz na elevação dos níveis de HDL (lipoproteína de alta densidade) e o ômega-3 reduz os níveis séricos de triglicerídeos. As informações presentes neste estudo poderão servir de base para a atualização de profissionais da saúde, contribuindo com a melhoria do desenvolvimento de estratégias que visem a adesão e manejo das dislipidemias.

Palavras-chave: dislipidemias, antidislipidêmicos, farmacologia.

\begin{abstract}
Dyslipidemias can be defined as changes in the lipid levels of the circulation, making the use of drugs essential to avoid the emergence of cardiovascular problems. The objective was to carry out a literature review on the main classes of drugs used in the treatment of dyslipidemia. It was a literature review in the SciELO, BVS, MedLine and PubMed databases. The bibliographic survey took place from January to June 2019. Statins inhibit the limiting step in cholesterol synthesis and are preferred drugs in hypercholesterolemia. Fibrates, in turn, are considered first-line drugs for hypertriglyceridemia, whereas bile acid sequestrants and ezetimibe are generally used in combination with other lipid-lowering agents in the treatment of hypercholesterolemia. Niacin modifies favorably all major lipid fractions and is the most effective drug in raising HDL (high density lipoprotein) levels and omega- 3 reduces serum triglyceride levels. The information presented
\end{abstract}


in this study may serve as a basis for the updating of health professionals, contributing to the improvement of the development of strategies aimed at the adherence and management of dyslipidemias.

Keywords: dyslipidemias, antidyslipidemics, pharmacology.

\section{Introdução}

As dislipidemias formam um grupo de distúrbios caracterizados por anormalidades quantitativas e/ou qualitativas nos triglicerídeos, colesterol e lipoproteínas de alta (HDL) e baixa densidade (LDL).

As alterações do perfil lipídico podem incluir colesterol total e triglicerídeos elevados, colesterol de lipoproteína de alta densidade baixo (HDL-c) e níveis elevados de colesterol de lipoproteína de baixa densidade (LDL-c). Assim, as dislipidemias podem ser classificadas como hiperlipidemias (elevados níveis de lipoproteínas) e hipolipidemias (baixos níveis plasmáticos de lipoproteínas) (SOCIEDADE BRASILEIRA DE CARDIOLOGIA, 2017).

$O$ tratamento das dislipidemias pode ser dividido em não medicamentoso e medicamentoso. O primeiro envolve basicamente mudanças nos hábitos e estilo de vida. Neste aspecto é essencial a perda de peso, a abolição do tabagismo e alcoolismo, a prática de exercícios físicos regularmente, melhorar os hábitos alimentares e o combate ao estresse e sedentarismo, enquanto que na segunda estratégia de tratamento tem-se os fármacos que são importantes aliados para o controle dos níveis séricos de colesterol, triglicerídeos e lipoproteínas (BRASIL, 2013; SOCEIDADE BRASILEIRA DE CARDIOLOGIA, 2017).

A concentração elevada de lipídeos séricos e os baixos níveis de HDL são fatores determinantes para a incidência de doenças como a aterosclerose e outras doenças relacionadas (BRUNTON et al., 2012). Por esse motivo, a abordagem terapêutica para o controle de níveis séricos de lipídeos engloba atualmente várias classes de medicamentos que, geralmente, são de uso contínuo e indefinido, uma vez que, apenas ajudam a corrigir o distúrbio do metabolismo, não levando a cura (SILVA et al., 2018; CONITEC, 2019). Desta forma, faz-se necessário o conhecimento atualizado por parte dos profissionais da saúde sobre a farmacoterapia das dislipidemias. 
Assim, o presente estudo teve como objetivo realizar uma revisão de literatura sobre as principais classes de fármacos utilizados para o tratamento das dislipidemias.

\section{Metodologia}

\subsection{Delineamento do estudo}

Tratou-se de uma revisão de literatura do tipo narrativa, uma vez que, a presente pesquisa apresenta-se como uma publicação que permite ampla descrição de um determinado assunto (ROTHER, 2007).

\subsection{Fontes de informação}

Foi realizada uma revisão da literatura nacional e internacional utilizando os bancos de dados Medline (Medical Literature Analysis and Retrieval System Online), PubMed, SciELO (Scientific Electronic Library Online) e BVS (Biblioteca Virtual em Saúde).

\subsection{Critérios de Inclusão e Exclusão}

A pesquisa foi realizada entre janeiro e junho de 2019 e incluiu artigos originais, artigos de revisão, escritos nas línguas inglesa, espanhola e portuguesa, publicados entre os anos de 2010 a 2019. Assim, foram selecionados aqueles que trouxessem informações relacionadas aos principais fármacos utilizados nas dislipidemias, farmacocinética e farmacodinâmica. Excluem-se artigos em outros idiomas e que não puderam ser acessados na íntegra.

\subsection{Termos de pesquisa}

Os seguintes termos de pesquisa (palavras-chaves e delimitadores) foram utilizados em várias combinações, através do operador booleano "and": 1) Dislipidemias; 2) Hipolipemiantes; 3) Lipídeos; 4) Dyslipidemia; 5) Hypolipidemic; 6) Lipids; 7) Dislipidemias; 8) Hipolipemiante; 9) Lípidos.

\section{Revisão de literatura}

\subsection{Estatinas}

As estatinas tratam-se de fármacos que podem ser divididos em naturais ou sintéticos, diferindo essencialmente em termos de potência, perfil 
farmacocinético, interação farmacológica e efeito indesejado relacionado à miotoxicidade. Contudo, atualmente, somente seis estatinas são empregadas clinicamente no Brasil, com destaque para Lovastatina, Pravastatina, Sinvastatina (derivado semi-sintético), Fluvastatina (Lescol) e, por fim, Atorvastatina e Rosuvastatina que são de estatinas sintéticas e enantiomericamente puras (CONITEC, 2019).

Estes fármacos são agentes hipolipemiantes que exercem os seus efeitos através da inibição da 3-hidroxi-3-metilglutaril CoA redutase (HMG-CoA redutase), bloqueando os primeiros passos da biossíntese de colesterol (figura 1). Estas substâncias agem por meio da afinidade com o sítio ativo da enzima, ocasionando em redução do colesterol tecidual e um consequente aumento na expressão dos receptores de LDL na superfície dos hepatócitos que, por sua vez, resulta em aumento da remoção do LDL do sangue e redução dos níveis plasmáticos de LDL-c. Contudo, esta inibição é reversível e competitiva com o substrato HMG-CoA. Ademais, as estatinas também apresentam uma sútil eficácia na concentração de HDL e redução dos triglicerídeos e VLDL (lipoproteínas de muito baixa densidade), atuando positivamente no controle do perfil lipídico (TOURNADRE, 2019).

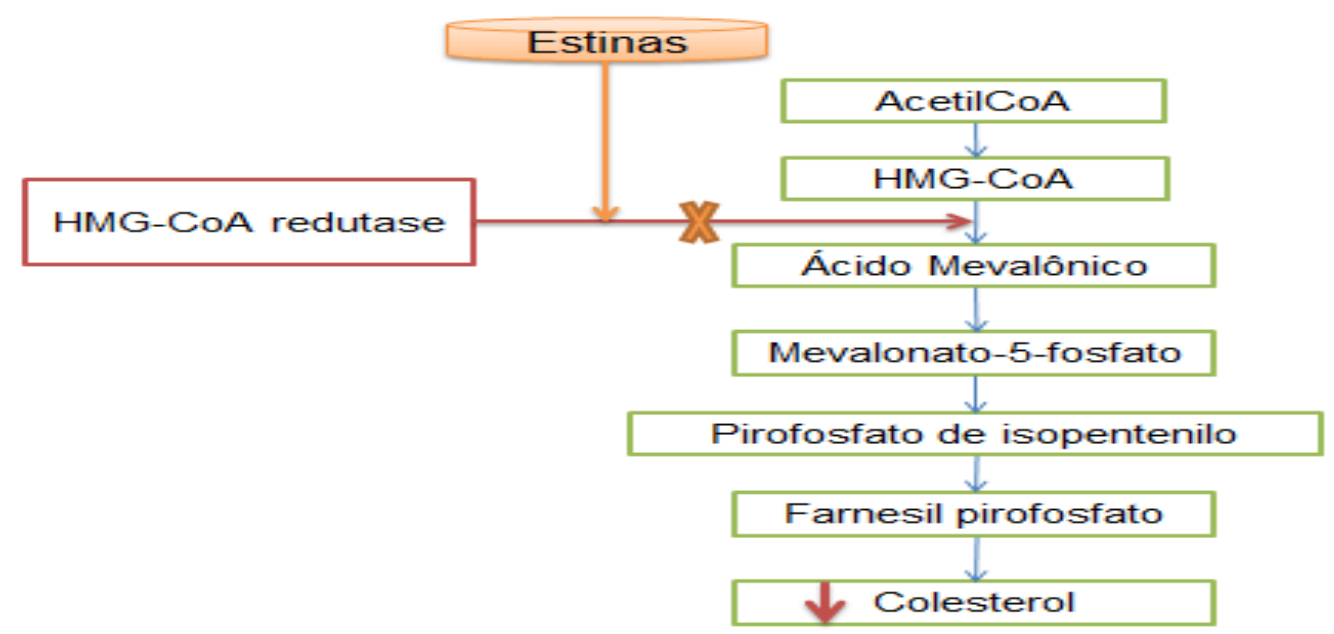

Figura 1. Mecanismo de ação das estatinas.

Fonte: Autoria própria, 2019.

A sinvastatina, é um pró-fármaco na forma de lactona derivado semisintético da lovastatina, que difere deste último apenas por ter em um grupo metil ao lado na cadeia (figura 2). Este fármaco foi inicialmente aprovado para comercialização na Suécia em 1988 e posteriormente em todo o mundo, 
seguido da fluvastatina (1994), atorvastatina (1997), cerivastatina (1998) e rosuvastatina (2003) (VARGAS; LIMBERGER, 2013).
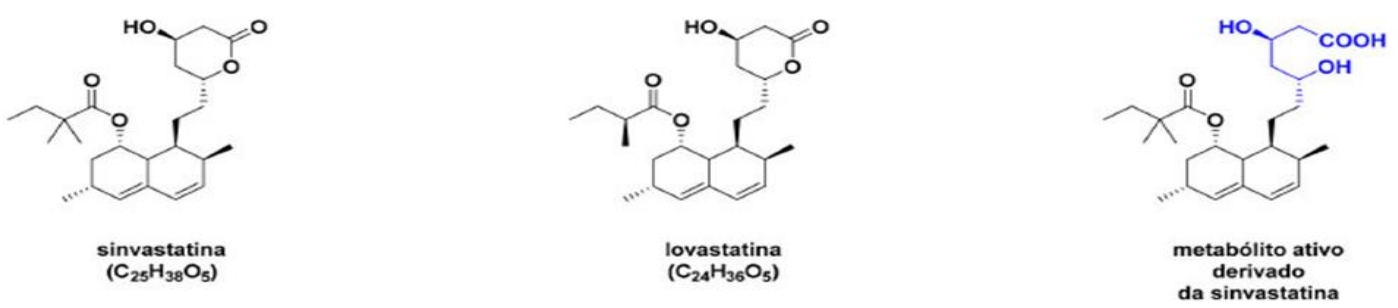

Figura 2. Fórmulas estruturais da sinvastatina, lovastatina e metabólito ativo.

Fonte: TAVARES, 2016.

As estatinas devem ser administradas por via oral, em dose única diária, preferencialmente à noite para os fármacos de curta meia-vida. Isso se justifica tanto por questões fisiológicas quanto farmacocinéticas, uma vez que a intensidade da síntese endógena de colesterol é mais elevada no período noturno (ritmo circadiano do organismo), momento em que a absorção intestinal de gorduras é mais baixa. Assim, para fármacos de meia vida mais curta, como a sinvastatina e a pravastatina, a administração noturna é recomendável para que se tenha seu pico de ação no período de maior atuação da HMG-CoA. Para as estatinas mais modernas, como atorvastatina e rosuvastatina, que apresentam meia-vida maior, a efetividade independente do momento de administração, podendo ser administradas em qualquer horário (SOCIEDADE BRASILEIRA DE CARDIOLOGIA, 2017).

No contexto da terapia medicamentosa as estatinas ganham destaque por constituírem a classe de agentes modificadores de lipídeos mais utilizados no tratamento das dislipidemias, apresentando eficácia comprovada na redução da mortalidade por doenças cardiovasculares e mostrando-se eficientes independentemente de sexo, idade ou nível de prevenção, seja primária ou secundária (PORTELA et al., 2014).

As estatinas promovem ainda vantagens que são independentes de sua função primária de redução dos níveis de colesterol sanguíneo, incluindo ação anti-inflamatória, antioxidante, modulação da pressão arterial, aumento da estabilidade de placa aterosclerótica e capacidade de proteção vascular que são conhecidos como seus efeitos pleiotrópicos (PORTELA et al., 2014). Além 
de apresentarem baixo custo e menos efeitos adversos, o que por sua vez, pode contribuir diretamente para o uso racional de medicamentos.

Apesar da incidência de efeitos adversos ser baixa, alguns pacientes, podem desenvolver toxicidade hepática e/ou muscular, em graus variados. São pouco conhecidos os mecanismos pelos quais as estatinas provocam hepatotoxicidade. Aproximadamente 1\% dos pacientes tratados apresenta elevação das transaminases na maioria das vezes transitória e/ou dose dependente. Contudo, doenças hepáticas preexistentes e o abuso de bebidas alcoólicas propiciam o aumento dessas enzimas. Além disso, os usuários desse tipo de fármaco podem apresentar hipercalemia e mialgias severas (LIU et al., 2019).

O efeito tóxico mais grave observado com o uso destes fármacos é a rabdomiólise que atinge cerca de 0,1 a 8,4/100.000 paciente por ano. O seu diagnóstico baseia-se no aumento das enzimas musculares, como a CPK (Creatinofosfoquinase), que pode apresentar valores dez vezes superior a normalidade (SOUICH; ROEDERER; DUFOUR, 2016).

O risco de miopatia aumenta com doses altas do medicamento e com o uso concomitante de outros fármacos, como fibratos, macrolídeos, varfarina, ciclosporina, antifúngicos imidazólicos e digoxina. Essa interação medicamentosa ocorre devido ao efeito inibidor destes fármacos no citocromo P450, que também é responsável pela metabolização das estatinas. Em associação agem aumentando a sua concentração sérica (CAUSEVIROMOSEVAC; SEMIZ, 2013).

Além disso, a mialgia e miosite são as causas mais comuns da não adesão à terapia com estatinas. Essas doenças envolvem tipicamente grandes grupos musculares corporais como os das pernas, ombros e porção superior dos braços, podendo ocasionar desconforto intenso e até perda da habilidade e força motora (SOUICH; ROEDERER; DUFOUR, 2016).

A insuficiência renal, por sua vez, ocorre em cada 0,3 a 0,9 casos por 1.000.000 de prescrições. Também tem sido descrita proteinúria, particularmente, com rosuvastatina na dose de $80 \mathrm{mg} / \mathrm{dia}$. No entanto, a doença renal crônica não contraindica o seu emprego, embora as doses devam ser ajustadas (SOUICH; ROEDERER; DUFOUR, 2016). 
As estatinas são contraindicadas para mulheres grávidas ou em fase de aleitamento, em pacientes com doença hepática e de elevações persistentes das transaminases hepáticas e de bilirrubinas, contudo podem ser usadas em pacientes renais crônicos (SANTIAGO, 2011).

\subsection{Fibratos}

Os fibratos ou derivados do ácido fíbrico são os medicamentos preferidos no tratamento da hipertrigliceridemia e apresentam uma importante ação no controle das dislipidemias mistas, tendo fenofibrato, ciprofibrato, etofibrato e bezafibrato como principais representantes (SOCIEDADE BRASILEIRA DE CARDIOLOGIA, 2017).

Estes fármacos atuam a partir da estimulação dos receptores nucleares ativados de proliferação dos peroxissomas-alfa, os conhecidos PPAR-a, que modulam a transcrição de múltiplos genes envolvidos no metabolismo lipídico, levando ao aumento da produção e da ação da lipase lipoproteica (LPL) que é responsável pelo catabolismo das lipoproteínas ricas em triglicerídeos. Parte deste efeito é devido à redução na síntese hepática de apo CIII, apolipoproteína que inibe a atividade desta enzima. Além disso, este mecanismo estimula a lipólise dos triglicerídeos das VLDL-colesterol (VLDLc) e dos quilomícrons. Dessa forma agem também na diminuição a síntese das VLDL e provocam, ainda, aumento na síntese das apolipoproteínas Al e All, fato que pode ajudar a explicar o aumento no HDL-c. Dessa forma, ao facilitar a degradação das VLDL em LDL, podem causar um discreto aumento no LDL-c. (CABRAL et al., 2017; SOCIEDADE BRASILEIRA DE CARDIOLOGIA, 2017). 


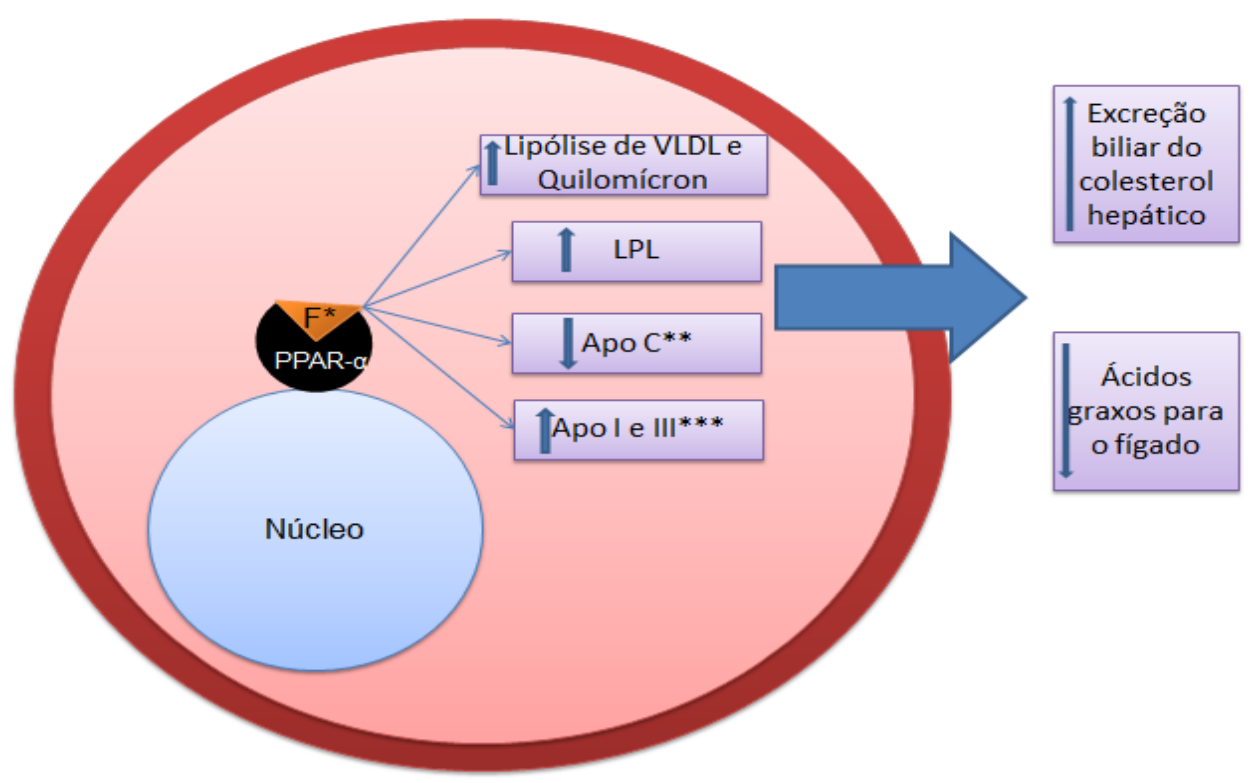

Figura 3. Mecanismo de ação dos fibratos.

*F=Fármaco; ** Apolipoproteína C; *** Apolipoproteina I e III. Fonte: Autoria própria, 2019.

Uma das associações comumente utilizadas na terapia hipolipemiante é a combinação das estatinas com os fibratos, a qual deve ser considerada quando a monoterapia ou a adição de outros fármacos (por exemplo, inibidores da absorção de colesterol, ômega-3, ácido nicotínico) não atinge as metas lipídicas ou é impraticável, também nos casos em que ocorre intolerância a doses mais elevadas das estatinas e nas situações de hipertrigliceridemia importante e redução de riscos pancreatite aguda (BRASIL, 2013).

Essa combinação pode ser realizada levando-se em conta o maior risco de reações adversas como rabdomiólise e miopatia. Ressalta-se que o risco de miopatia é maior para combinação de estatinas com a gemfibrozila do que com o bezafibrato ou fenofibrato, provavelmente por inibir a glucuronidação desta e acentuar a desestabilização da membrana celular, sendo que o risco de miopatia é mais acentuado em diabéticos, renais crônicos, hipotireóideos e idosos (SOCIEDADE BRASILIERA DE CARDIOLOGIA, 2017).

\subsection{Sequestradores de ácidos biliares}

Os sequestradores de ácidos biliares (AC) são cloretos de resina de troca aniônica, não absorvíveis no trato gastrintestinal. Seu mecanismo baseia-se na ligação a ácidos biliares o que permite a formação de um complexo fármaco- 
ácido biliar, promovendo, a eliminação dos mesmos por meio das fezes, ocasionando em menor reabsorção dos ácidos biliares (figura 3). Isso faz com que o organismo observe o déficit de AC retornando ao fígado, resultando em maior conversão hepática de colesterol nesses compostos e consequente redução da biodisponibilidade de colesterol nos hepatócitos (GRILO, 2012)

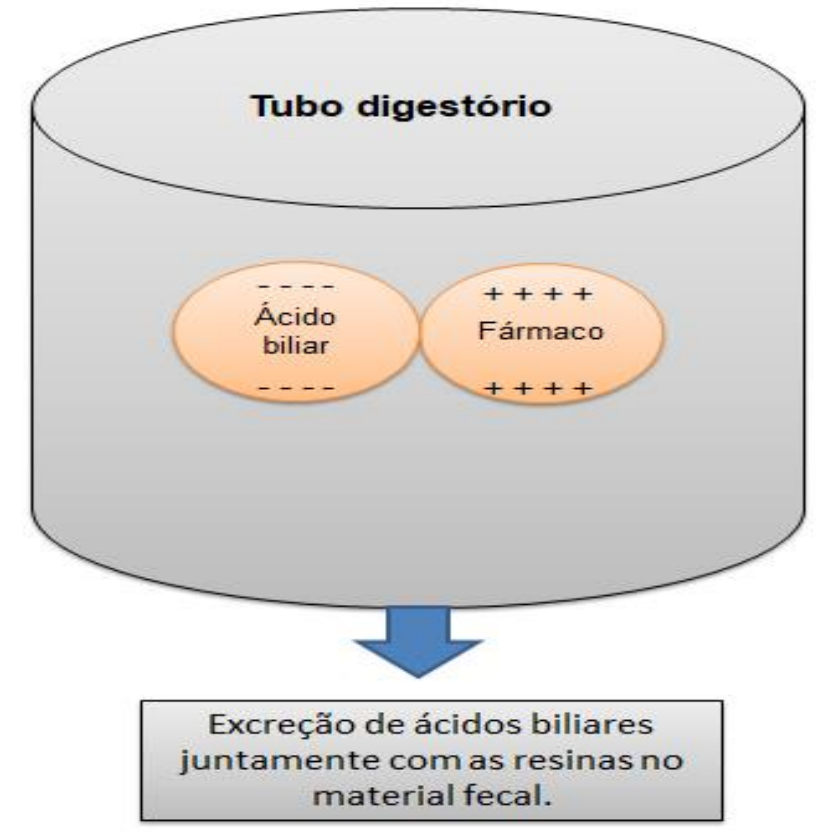

Figura 3. Mecanismo de ação dos sequestradores de ácidos biliares.

Fonte: Autoria própria, 2019.

Devido sua baixa tolerabilidade, as resinas são considerados fármacos de segunda linha para o tratamento da hipercolesterolemia, contudo devido a grande segurança que apresentam, podem ser utilizados em menores de oito anos de idade (CABRAL et al., 2017).

O uso prolongado desses fármacos podem causar hipoprotrombinemia em consequência da deficiência de vitamina $K$, prevenida ou revertida pela administração oral ou parenteral dessa vitamina. Entre os efeitos bioquímicos, citam-se discreto aumento dos triglicerídeos, de fosfatase alcalina e transaminases, sendo que 0 seu uso deve ser evitado se houver hipertrigliceridemia. A colestiramina, mais especificamente, é indicada em casos de hipercolesterolemia discreta ou moderada, consequente a causas ambientais ou genéticas (de caráter heterozigótico), não atuando na hipercolesterolemia familiar homozigótica devido à impossibilidade de expressão de receptores B-E. Pode ser administrada a pacientes de qualquer 
idade, ressalvando que os idosos a toleram com menos facilidade devido aos efeitos colaterais. Entretanto, é o único fármaco permitido para crianças e adolescentes que apresentam hipercolesterolemia resistente às medidas de restrição alimentar (SOCIEDADE BRASILEIRA DE CARDIOLOGIA, 2017).

\subsection{Ezetimiba}

Em função dos efeitos gastrointestinais e interferência na absorção de outros medicamentos, causados pelas resinas sequestrantes de ácidos biliares, a ezetimiba apresenta-se como uma possível opção para pacientes impossibilitados de fazer o uso de resinas ou ainda, para aqueles que não apresentem adesão ao tratamento.

A ezetimiba apresenta mecanismo de ação único, agindo através da inibição do transportador de esterol Niemann-Pick C1-Like 1 (NPC1L1) que trata-se de uma proteína transmembrana localizada na superfície dos enterócitos. Assim, o fármaco fica localizado na borda em escova dos enterócitos do intestino delgado impedindo a absorção de esteróis (figura 4). Isto ocasiona em redução do transporte de colesterol do intestino para o fígado, fazendo com que haja um déficit nos estoques de colesterol hepático e considerável aumento depuração do colesterol sanguíneo devido o aumento da expressão hepática de receptores LDL (GRILO, 2012; BRASIL, 2015).

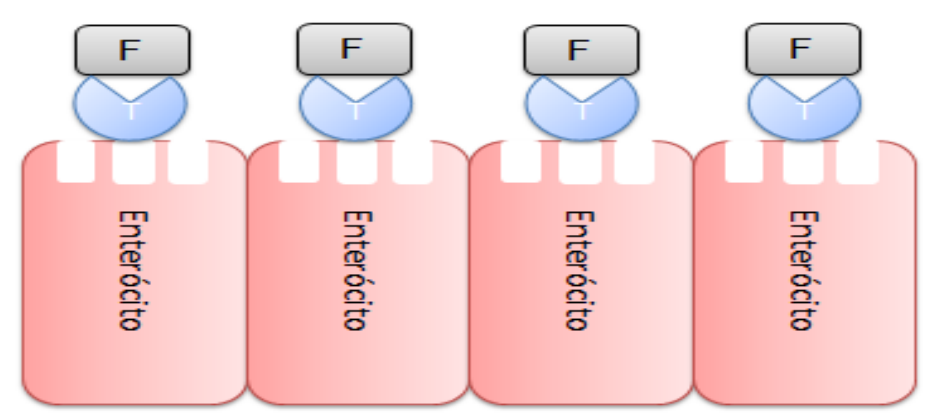

Figura 4. Mecanismo de ação, simplificado, de ezetimiba.

F=Fármaco, $\mathrm{T}=$ Transportador $\mathrm{E}=$ Esterol .

Fonte: Autoria própria, 2019.

Este fármaco tem a propriedade de ser rapidamente captado pelas células intestinais após ingestão oral quando passa a sofrer processo de glucuronidação na parede intestinal originando um metabólito glucuronídeo 
farmacologicamente ativo. Nesta forma, é absorvido e atinge concentração plasmática máxima cerca de 1 hora após, quando então é captado pelo fígado e retorna à luz intestinal onde vai permanecer na borda vilosa dos enterócitos, bloqueando a captação dos esteróis. Sua meia vida plasmática apresenta uma variabilidade de duração efetiva idade-dependente, alcançando maior permanência plasmática nos indivíduos mais idosos. Apresenta discreto aumento da sua absorção quando ingerida conjuntamente com a alimentação (CABRAL et al., 2017).

A associação estatina e ezetimiba constitui uma interessante opção terapêutica no tratamento das dislipidemias, por apresentar um ótimo perfil de segurança e eficácia. Quando a combinação de baixas doses da estatina com ezetimiba é comparada com altas doses da estatina isoladamente, observa-se que as duas formas de tratamento são equivalentes em eficácia e na redução LDL-C, com a vantagem de apresentar menos efeitos adversos. Contudo, seu uso pode provocar distúrbios gastrointestinais, dores de cabeça, fadiga, raramente mialgia e discretas elevações das transaminases (SERRANO, 2010; CORREIA, 2016).

\subsection{Derivados do ácido nicotínico (Niacina)}

O ácido nicotínico ou niacina é um vitamina solúvel e foi o primeiro agente hipolipemiante usado para o tratamento da dislipidemia (CABRAL et al., 2017).

Mesmo o mecanismo de ação da niacina não sendo ainda bem estabelecido, existem evidências que atue inibindo o transporte dos ácidos graxos livres dos tecidos periféricos ao fígado; desta forma, reduz eficazmente a síntese hepática de triglicerídeos, resultando na diminuição da produção de lipoproteínas contendo apo-B. Niacina diminui também o catabolismo das apoAl e reduz os níveis de triglicerídeos (20-50\%) e de LDL colesterol (5-25\%), além de aumentar o HDL-c em 15-35\%. Além disso, esse fármaco ainda modifica favoravelmente todas as principais frações lipídicas, sendo o único agente hipolipemiante que diminui a lipoproteína (a), e o fármaco mais eficaz na elevação dos níveis de HDL-c (BRASIL, 2015).

Existem três formulações do ácido nicotínico: de liberação imediata ou cristalina, liberação intermediária ou prolongada e a forma de liberação lenta. A 
niacina de liberação imediata é rapidamente absorvida e excretada. Atinge pico sérico cerca de 30 a 60 minutos de sua ingestão e sua meia vida metabólica é de uma hora. A niacina de liberação lenta, também conhecida como de liberação controlada ou sustentada, se caracteriza por apresentar seu tempo de dissolução superior a 12 horas. A niacina de liberação intermediária ou estendida é absorvida em um período de 8 a 12 horas, intermediário entre a niacina cristalina e a lenta (MARIA; MOREIRA, 2011).

Em relação aos efeitos colaterais, o mais evidente é o "flushing", ou seja, calor e rubor geralmente acompanhado de prurido motivado pela liberação de prostaglandinas durante a formação do ácido nicotinúrico, afetando principalmente o rosto, pescoço e parte superior do tronco, mas por vezes, se prolonga para a parte inferior do corpo. Além disso, pode haver sugimento de erupção cutânea, hipotensão e tonturas. Estes efeitos adversos podem ser melhorados quando a niacina é ingerida juntamente com as refeições, evitando a ingestão concomitante de álcool, alimentos condimentados e bebidas quentes (BRASIL, 2015).

Além disso, tem-se observado que suplementos contendo as vitaminas niacina (B3) e piridoxina (B6) podem promover a redução dos níveis de colesterol total e um aumento na concentração do colesterol HDL (LAMEIRO, et al, 2012; BRASIL, 2015).

\section{6 Ômega-3}

Os ácidos graxos essenciais, linoléico e a-linolênico, são aqueles que não podem ser sintetizados pelos tecidos dos mamíferos e devem necessariamente ser obtidos a partir da dieta por ingestão de peixes de água fria, nozes, sementes de linhaça e óleos vegetais (EGíDIO; SOUSA JÚNIOR; MENEZES, 2016).

O ômega-3, mais especificamente, é um ácido graxo (AG) essencial, também denominado a-linolênico, composto de 18 átomos de carbono e 3 duplas ligações (C18:3) e está presente em alguns óleos vegetais (óleos de soja, canola e linhaça), apresentando sua primeira dupla ligação entre o terceiro e o quarto átomos de carbono, contando-se a partir do grupamento metil (SILVEIRA; MOREIRA, 2017). 
Dentre os ácidos graxos poliinsaturados ômega-3 de cadeia longa (superior a 18 carbonos), tem-se destaque para o ácido eicosapentaenóico (EPA; C20:5) e o ácido docosahexaenóico (DHA; C22:6). Estes estão naturalmente presentes em produtos de origem marinha. $\mathrm{Na}$ espécie humana, os tecidos capazes de biossintetizar EPA e DHA são o fígado, as gônadas, e em menor escala, o cérebro e o tecido adiposo, e o fazem a partir do precursor ácido alfa-linolênico, através de sistemas enzimáticos de alongamento e dessaturação, ainda que a velocidade desta transformação seja muito lenta, principalmente quando a dieta é rica em ácido linoléico, que compete pelas mesmas dessaturases (SILVEIRA; MOREIRA, 2017).

Grande parte dos efeitos dos ácidos graxos poliinsaturados sobre 0 metabolismo, a diferenciação e o crescimento celular ocorre por meio de alterações no padrão de expressão dos genes responsivos aos Receptores Ativados por Proliferadores de Peroxissomos (PPAR). Os PPAR são receptores de hormônios esteróides que atuam como fatores de transcrição ativados por ligantes. Estes receptores ocorrem em três diferentes isoformas ( $\alpha, \beta / \delta$ e $\gamma$ ), que exibem padrões de expressão tecido-específicos e modulam a transcrição de diferentes genes envolvidos na homeostase e no metabolismo lipídico. Em geral, todos os AG ômega 3 (n-3) e ômega 6 (n-6) ativam as três isoformas dos receptores nucleares PPARa, $\beta$ e $\gamma$, mas com diferentes afinidades pelos subtipos (RAPOSO, 2010).

O ômega 3 (n-3) é efetivo na redução dos níveis plasmáticos de triglicerídeos visto que a ativação do PPAR- $\alpha$ aumenta a lipólise intravascular e o clearance das partículas ricas em triglicerídeos. Reduzem os triglicerídeos por diminuir a produção de VLDL no fígado. A dose mínima recomendada é de $4 \mathrm{~g} / \mathrm{dia}$ sendo utilizado como adjuvantes aos fibratos na terapia das hipertrigliceridemias ou em substituição a esses em pacientes intolerantes (RAPOSO, 2010; SIMÃO et al., 2010).

Os ácido eicosapentaenóico e o ácido docosaexaenóico apresentam efeitos adversos relativamente comuns, estando associados ao sistema gastrointestinal. Além disso, relata-se a interação destes com anticoagulantes levando a potencialização dos seus efeitos. Contudo, os ômega-3 devem ser utilizados com precaução em pacientes com hipercolesterolemia, uma vez que, 
tendem a aumentar o colesterol LDL nestes e também em diabéticos, em que há uma piora do controle da glicose (SOCIEDADE BRASILEIRA DE CARDIOLOGIA, 2017).

Por fim, torna-se importante enfatizar que o estilo de vida é determinante no sucesso do tratamento de dislipidemias, sendo portanto de extrema importância que o profissional farmacêutico oriente o paciente antes, durante ou após a dispensação, com 0 intuito de possibilitar o autocuidado e conhecimento para o paciente sobre as dislipidemias, os medicamentos utilizados e medidas não farmacológicas que podem ser aplicadas.

\section{Conclusão}

As classes de medicamentos empregadas no tratamento das dislipidemias são: estatinas, fibratos, sequestrantes de ácidos biliares, ezetimiba, niacina e ômega-3.

As estatinas, sequestradores de ácidos biliares e ezetimida mostraramse importantes no tratamento da hipercolesterolemia. Os fibratos, por sua vez, são considerados medicamentos de primeira escolha para a hipertrigliceridemia. A niacina é o fármaco mais eficaz na elevação dos níveis de HDL e o ômega-3 reduz os níveis séricos de triglicerídeos.

Dessa forma, torna-se importante ressaltar que as informações presentes neste estudo poderão servir de base para a atualização de profissionais da saúde, contribuindo com a melhoria do desenvolvimento de estratégias que visem a adesão e manejo das dislipidemias, explorando o potencial de atuação do profissional farmacêutico no cuidado direto ao paciente, preenchendo a lacuna existente entre o benefício potencial do arsenal terapêutico e o seu real valor na redução da morbimortalidade relacionada ao uso dos medicamentos.

\section{Referências}

BRASIL. Ministério da Saúde. Dislipidemia: prevenção de eventos cardiovasculares e pancreatite. 2013. Disponível em:< http://portalarquivos2.saude.gov.br/images/pdf/2014/abril/02/pcdt-dislipidemia-livro2013.pdf>. Acesso em: 05 mai. 2019. 
BRASIL. Ministério da Saúde. Metri (ácido nicotínico). 2015. Disponível em: < http://www.anvisa.gov.br/datavisa/fila_bula/frmVisualizarBula.asp?pNuTransacao $=138$ 48402016\&pldAnexo=3166724 >05 mai. 2019. Acesso em: 05 mai. 2019.

BRUNTON Laurence $L$ et al. As bases farmacológicas da terapêutica. Rio de Janeiro: Editora Mc Graw Hill, 2012.

CABRAL, Mateus Costa. et al. Farmacologia da dislipidemia e aterosclerose. Revista Científica Fagoc Saúde, v.3, p.73-79, 2017.

CAUSEVIC-RAMOSEVAC, Anida.; Sabina, SEMIZ. Drug interaction with statins. Acta Pharmaceutica, v.63, n.3 p.277-293, 2013.

CONITEC. Comissão Nacional de Incorporação de Tecnologia no SUS. Protocolo Clínico e Diretrizes Terapêuticas Dislipidemia: prevenção de eventos cardiovasculares e pancreatite. 2019. Disponível em:< http://conitec.gov.br/images/Consultas/Relatorios/2019/Relatorio_PCDT_Dislipidemia_ CP04_2019.pdf>. Acesso em: 01 mar. 2019.

CORREIA, Luis C. L. Ezetimibe: significado clínico e científico do estudo improve-IT. Arquivos Brasileiros de Cardiologia, v.106, n.3, p.247-249, 2016.

EGÍDIO, Elvis de Souza.; SOUSA JÚNIOR, Jair Rodrigues.; MENEZES, Maria Emília da Silva. Alimentos funcionais: uma revisão. Educação Ciência e Saúde, v.3, n.1, p.11-29, 2016.

GRILO, Maria João Costa. Terapêutica Farmacológica das dislipidemias. 2012. 52 f. Dissertação (Mestrado em Ciências Farmacêuticas) - Universidade Fernando Pessoa, Porto, 2012.

LIU, Aimei. et al. Statins: adverse reactions, oxidative stress and metabolic interactions. Pharmacology \& Therapeutics, v. 195, p.54-84, 2019.

MARIA, Carlos Alberto Bastos de.; MOREIRA, Ricardo Felipe Alves. A intrigante bioquímica da niacina: uma revisão crítica. Química Nova, v. 34, n.10, p.1739-1752, 2011. 
PORTELA, Alyne da Silva. et al. Estatinas x ácido lipóico na prevenção e tratamento das doenças cardiovasculares. Revista de Ciências Farmacêuticas Básica e Aplicada, v.35, n.1, p.9-15, 2014.

RAPOSO, Helena Fonseca. Efeito dos ácidos graxos n-3 e n-6 na expressão de genes do metabolismo de lipídeos e risco de aterosclerose. Revista de Nutrição, v.23, n.5, p.871-879, 2010.

ROTHER, Edna Terezinha. Revisão sistemática x revisão narrativa. Acta Paulista de Enfermagem, São Paulo, v.20, n.2, 2007.

SANTIAGO, Marcos A. da Costa. Estatinas - Efeitos tóxicos e novas aplicações. 2011. 86 f. Dissertação (Mestrado em Ciências Farmacêuticas) - Universidade Fernando Pessoa, Porto, 2011.

SERRANO, Cumplido A. Indicaciones de loshipolipemiantes. Información terapéutica del Sistema Nacional de Salud. v.34, n. 2, p.41-48, 2010.

SILVA, Ederson Aparecido. et al. O uso das estatinas no tratamento da dislipidemia e o mecanismo da biossíntese do colesterol. Revista Científica FAEMA. v.9, p.597-602, 2018.

SILVEIRA, Deuclides Caetano Soares Rodrigues.; MOREIRA, Elma Eliane. Efeitos da utilização do ômega-3 no processo de envelhecimento: uma revisão. Revista Científica FacMais, v.8, n.1, p.137-155, 2017.

SIMAO, Andréa Name Colado. et al. Efeito dos ácidos graxos $n$-3 no perfil glicêmico e lipídico, no estresse oxidativo e na capacidade antioxidante total de pacientes com síndrome metabólica. Arquivos Brasileiros Endocrinologia e Metabologia, v. 54, $n$. 5, p. 463-469, 2010.

SOCIEDADE BRASILEIRA DE CADIOLOGIA. Atualização da Diretriz Brasileira de Dislipidemias e Prevenção da Aterosclerose. Arquivos Brasileiros de Cardiologia, $v$. 109, supl.1, p.1-76, 2017.

SOUICH, Patrick du.; ROEDERER, Ghislaine.; DUFOUR, Robert. Myotoxicity of statins: Mechanism of action. Pharmacology \& Therapeutics, v.175, n. p.1-16, 2017. 
TAVARES, Maurício Temotheo et al. Entendendo o processo químico de bioativação da sinvastatina por métodos experimentais e computacionais: uma aula prática. Química Nova, v. 39, n. 4, p. 502-506, 2016.

TOURNADRE, Anne. Statins, myalgia, and rhabdomyolysis. Joint Bone Spide, v.87, n.1, p.37-42, 2019.

VARGAS, Thayne Castelan.; LIMBERGER, Jane Beatriz. Tratamento farmacológico com estatinas: uma revisão sistemática. Disciplinarum Scientia, v.14, n.2, p.175-187, 2013. 